\title{
Introduction
}

\section{The Nature and Needs of Americans with Emerging Disabilities}

\author{
Lynn C. Koch ${ }^{\mathrm{a}, *}$ and Phillip D. Rumrill, Jr. ${ }^{\mathrm{b}}$ \\ ${ }^{a}$ University of Arkansas, Fayetteville, AR, USA \\ ${ }^{\mathrm{b}}$ Kent State University, Kent, OH, USA
}

Revised/Accepted September 2016

The number of individuals with disabilities continues to grow as a result of contemporary societal trends such as rapid medical advances in diagnostics and treatment, globalization, civil rights legislation that has broadened the concept of disability, violence, poverty, climate change, and the aging of the American populace (Koch \& Rumrill, 2016). Included in the expanding constellation of disabilities are conditions that often have unknown etiologies, are medically debated, are less clearly defined by law and public policy, have higher rates of incidence in already marginalized populations, and are often underestimated in terms of the severity of their symptoms and the substantial impact of these symptoms on individuals' ability to actively participate in meaningful life activities such as employment (Fox \& Kim, 2004; Koch, Rumrill \& Conyers, 2012; Nary, White, Budde \& Vo, 2004). The umbrella term used for these conditions is emerging disabilities. Emerging disabilities result from diseases or health conditions that are either (a) recently recognized and increasing in prevalence in a population (e.g., Ehlers-Danlos Syndrome [EDS]) or (b) established conditions that are increasing in prevalence in a population or in specific segments of a population (e.g., autism spectrum disorder [ASD], chronic pain, attention deficit disorder

\footnotetext{
*Address for correspondence: Lynn C. Koch, Ph.D., CRC, University of Arkansas, Rehabilitation, Human Resources, and Communication Disorders, Fayetteville, AR, USA. Tel.: +1 479 575 5824; E-mail: 1ckoch@uark.edu.
}

[ADD], multiple sclerosis [MS]; human immunodeficiency virus [HIV]; Fujiura, 2001).

Nary et al. (2004) observed that people with emerging disabilities often exhibit less apparent physical disability, even though their conditions can be severe and negatively impact multiple functional domains. Ribet (2011, p. 161) expanded on the definition of emerging disabilities, defining these as "a pattern of burgeoning mental and physical conditions which correlates, often strongly, with poverty and various forms of social and political insubordination." Nary et al. and Ribet noted that emerging disabilities are linked to social inequities based on demographic characteristics of individuals such as race, ethnicity, gender, sexual orientation, gender identity, age, and socioeconomic status as well as the simultaneous interaction of these characteristics. These authors also pointed out that emerging disabilities would not be present or as severe if they did not occur in a context of systemic inequities and social injustices resulting from violence, limited access to health care, poor nutrition, inadequate housing, employment discrimination, labor exploitation, exposure to environmental hazards, institutionalization, and incarceration. Fox and Kim (2004) also distinguished emerging disabilities from traditional disabilities in terms of social and environmental factors that contribute to their onset, presentation, severity, and consequences. They noted that, in comparison to people with traditional disabilities, people 
with emerging disabilities experience greater barriers to full inclusion in society; therefore, consideration of social and environmental determinants of disability such as systemic inequities and social injustices should be a fundamental component of the vocational rehabilitation (VR) planning process.

Because many individuals with emerging disabilities experience symptoms with no detectable pathological basis, they must often contend with diagnostic uncertainties and consult multiple medical specialists who are perplexed by their reported symptoms (Koch et al., 2012). In such cases, physicians may conclude that these individuals have medically unexplained symptoms. Medically unexplained symptoms are those "for which conventional biomedical explanation could not be found on routine examination or investigations" (Nimnuan, Hotopf \& Wessely, 2001, p. 366). In some cases, medically unexplained symptoms may be mild and transient, whereas in other cases they are severe, ongoing, and debilitating. Medically unexplained symptoms challenge the conventional biomedical model of disease that focuses on definitive diagnosis and cure and fails to acknowledge the complexity of biopsychosocial factors in the onset and presentation of symptoms (Day, Thorn \& Burns, 2012). Physicians' inability to diagnose symptoms can lead to blaming patients, labeling symptoms as psychosomatic, and invalidating their illness experiences rather than examining shortcomings inherent in the biomedical approach to medicine (Raymond \& Brown, 2000; Van Houdenhove \& Luyten, 2006). The lack of a definitive medical diagnosis can prevent individuals from qualifying for specific civil rights protections, disability entitlements, and VR services. Indeed, being told that one's symptoms are medically unexplained or psychosomatic is a far too common experience for individuals with emerging disabilities.

In addition to recently recognized conditions, established conditions that are growing in incidence, and conditions that are difficult to diagnose, another emerging disability population consists of individuals with rare diseases or disorders. Rare disorders are those that affect fewer than 5,000 people in the United States (National Organization for Rare Disorders [NORD, 2016]). According to NORD, approximately 7,000 diseases in the United States are considered to be rare disorders. Individuals with these conditions are largely underserved by both health care and VR systems, in part due to their small numbers and in part due to their marginalized status (Koch et al., 2012). Rare disorders are extremely difficult to diagnose, and specialists and diagnosticians who are knowledgeable about these low-incidence conditions are often limited in number. Individuals with rare disorders, unlike those with medically established conditions, have fewer available resources to cope with their conditions, and, because of the low incidence of rare disorders, individuals with these disorders are not a potent political group (Koch et al., 2012). Finally, because research on rare disorders has primarily focused on the etiology of these conditions and finding cures, there is limited attention directed toward the vocational impact of these conditions and VR interventions that could improve employment outcomes for individuals with rare disorders.

In examining emerging disability populations, emerging causes of disability (e.g., violence, lifestyle factors, aging, poverty, climate change) must be taken into account. These causal factors intersect with disability, demographic characteristics of the individual, and social inequities to create unique challenges that must be addressed in VR plans if individuals with emerging disabilities are to achieve their employment goals. Finally, in considering emerging disability populations, we include those populations that are increasingly seeking services from VR professionals across rehabilitation settings as well as within specific settings. For example, rehabilitation counselors working in vocational settings can anticipate serving growing numbers of older Americans with disabilities as members of the Baby Boom generation continue to age (Wickert, Dresden \& Rumrill, 2013). As another example, it has been estimated that 50,000 youths with ASD reach adulthood each year (Shattuck, Narendorf, Cooper, Sterzing, Wagner \& Taylor, 2012), and ASD advocates have raised concerns about the capacity of adult service providers to adequately address the housing, independent living, educational, and employment needs of this rapidly increasing population of adults with disabilities.

Individuals with emerging disabilities represent a vastly underserved population who are often in dire need of VR services. These individuals stand to benefit from the same VR services as those with traditional disabilities (i.e., those that are medically recognized and well-understood by rehabilitation, medical, and healthcare professionals; Fox \& Kim, 2004; Koch et al., 2012). However, they often experience barriers (e.g., mixed messages received from physicians regarding their capacity to work, lack of identification as persons with disabilities, unawareness of the availability of services, lack of knowledge on the part of VR professionals regarding the medical 
and vocational aspects of their conditions, exclusionary eligibility criteria) to accessing and receiving VR services that will improve their employment outcomes.

Clearly, contemporary VR professionals will be continuously challenged to acquire new knowledge, skill sets, and competencies to improve employment outcomes and career pathways for people with emerging disabilities. With that in mind, this special issue of the Journal of Vocational Rehabilitation was developed to examine considerations for providing responsive VR services to individuals with a variety of disabilities and chronic illnesses that are included under the umbrella of emerging disabilities. Contributors to this special issue have examined important issues related to the provision of VR services to individuals with a variety of emerging disabilities including multiple sclerosis, HIV/AIDS, learning disabilities, ASD, ADHD, disabilities related to aging, and rare disorders.

Taken together, the articles contained in this special issue reflect the breadth and depth of vocational issues facing individuals with various emerging disabilities as well as important considerations regarding their VR service needs. It is our hope that this issue presents content that will aid VR professionals in better understanding the medical, psychosocial, and vocational implications of these emerging disabilities and developing evidence-based practices to promote employment opportunities and long-term career success for individuals with these emerging disabilities.

We wish to thank the authors who contributed articles to this issue. Our gratitude also goes to the Journal of Vocational Rehabilitation's Editor, Dr. Paul Wehman, for the opportunity to complete this work.

\section{References}

Day, M. A., Thorn, B. E., \& Burns, J. W. (2012). The continuing evolution of biopsychosocial interventions for chronic pain. Journal of Cognitive Psychotherapy, 26(2), 114-129.
Fox, M. H., \& Kim, K. (2004). Understanding emerging disabilities. Disability \& Society, 19(4), 323-337.

Fujiura, G. T. (2001). Emerging trends in disability. Population Today, 29(6), 9-10.

Koch, L. C., \& Rumrill, P. D. (2016). Rehabilitation counseling and emerging disabilities: Medical, psychosocial, and vocational aspects. NY: Springer Publishing Company.

Koch, L. C., Rumrill, P. D, \& Conyers, L. M. (2012). The nature and needs of people with emerging disabilities. In M. Bishop, P. Rumrill, \& P. Toriello (Eds.), New directions in rehabilitation counseling: Creative responses to professional, clinical, and educational challenges (pp. 115-139). Springfield IL: Charles C. Thomas.

Nary, D. E., White, G. W., Budde, J. F., \& Vo, H. Y. (2004). Identifying the employment and vocational rehabilitation concerns of people with traditional and emerging disabilities. Journal of Vocational Rehabilitation, 20(1), 71-77.

National Organization of Rare Disorders (2016). Rare disorder information. Retrieved from http://rarediseases.org/ for-patients-and-families/information-resources/rare-diseaseinformation/

Nimnuan, C., Hotopf, M., \& Wessely, S. (2001). Medically unexplained symptoms: An epidemiological study in seven specialties. Journal of Psychosomatic Research, 51(1), 361367.

Raymond, M. C., \& Brown, J. B. (2000). Experience of fibromyalgia: Qualitative study. Canadian Family Physician, 46, 1100-1106.

Ribet, B. (2011). Emergent Disability and the Limits of Equality: A Critical Reading of the UN Convention on the Rights of Persons with Disabilities. Yale Human Rights and Development Law Journal, 14.

Shattuck, P. T., Narendorf, S. C., Cooper, B., Sterzing, P. R., Wagner, M., \& Taylor, J. L. (2012). Postsecondary education and employment among youth with an autism spectrum disorder. Pediatrics, peds-2011.

Van Houdenhove, B., \& Luyten, P. (2006). Stress, depression and fibromyalgia. Acta Neurologica Belgica, 106(4), 149.

Wickert, K. M., Dresden, D. S., \& Rumrill, P. D., Jr. (2013). The sandwich generation's guide to eldercare. NY: DemosHealth. 